\title{
Review
}

\section{Inverse Problems in the Food Industry}

\author{
Tomowo MiHORI \\ Midori-ku Ohji-cho, 1229-62, Chiba 267-0065, Japan
}

Received May 31, 2004; Accepted October 29, 2004

\begin{abstract}
An issue often imposed on engineering is said to be essentially an inverse problem. Not to calculate the lethality of microorganisms in a packaged food under a given heat sterilization condition, but to find a heating condition to realize a demanded lethality is an example of inverse analysis. Using this technique, a problem that cannot be challenged through a traditional deterministic process can be solved. This article surveys the research related to the way of on-line control to appropriately manage the required heat sterilization effect on a product without knowing its thermal properties and to the manner of controlling a freezing system of a food that has thermal properties intensely dependent on temperature.
\end{abstract}

Keywords: inverse problem, sterilization, freezing, optimization, on line control

\section{Introduction}

The problem of analyzing the performance of an object of which the shape is specified requires solving a differential equation with appropriate initial/boundary conditions and is called a "direct problem". This direct problem cannot be solved, if one lacks such information as the shape, the boundary conditions or the physical properties of the object. When the physical quantities which correspond to the solution of this differential equation are observed, the initially lacked information will be known. The analyzing technique used from such a viewpoint is referred to as an inverse problem.

Groetsch (1993) pointed out that the history of the inverse problem goes back more than two thousand years. Finding the shape of a slip guide, which an object reaches earliest from a higher position to a lower position along the guide, is an inverse problem. (See section 2.1). In the 17 th century, Jakob Bernoulli found a beginning of the "variational principle" in relation to this mechanical problem. His younger brother Johann called it "functio" (1714). After that, in the 19th century Leonhard Euler, Joseph Louis Lagrange, and Adrien Marie Legendre studied a similar problem.

The traditional direct problem of a given canned food had been to find a sterilization factor, under the condition of a time/temperature profile of the retort. At first, Hildenbrand (1980) applied an inverse problem of a wide sense definition to compute the time/temperature history which minimizes a nutritional loss under the condition to realize a required sterilization value, using the variational principle. The required sterilization value is called "constraints" and the destruction quantity of the nutrition to be minimized is called the "performance function". In either a direct or an inverse problem, the thermal properties of the food and a heat transfer coefficient at the outer surface of the product must be correctly given, as well as the " $z$ value" and the " $D$ value" of a specially selected microorganism. At symposium on the optimization problem was presented by the IFT in June 1981. That out-view, which is an excellent guide for newcomers, was carried in the edition of Food Science Technology published in July 1982.

The variational principle is the basis on which to establish the FEM (finite element method) and the BEM (boundary element method). As is well known, FEM and BEM have become powerful implements to solve a wide class of direct problems. There are few people who call this technique inverse. The definition of an inverse problem in narrow sense is as follows.

(1) to estimate the causes of a phenomenon from the observed effect.

(2) to estimate some necessary conditions to solve a differential equation using the observed physical quantity which corresponds to the solution of this differential equation.

(3) to solve an "integral equation".

The integral equation that appears in the history of mathematics is the work of Abel $(1823,1826)$. (See section 2.2). Ferrari and Tricomi (1962) compiled the manner of designing a shape to realize a performance requiring nozzles or airfoils. This should pay attention to being used as an actual designing tool at the factory of an aircraft industry and not ended in a theoretical discussion in a salon. The problem to find the shape of an aerofoil of which the pressure distribution is given along the sectional profile of aerofoil has been of interest as an "indirect problem".

As soon as powerful computers become less expensive, inverse analysis will be applied in various fields, e.g. image diagnosis for medical, geological, remote sensing, astronomy, economics and so on. Most measurement methods for thermal properties of materials are established 
as an IHCP (inverse heat conduction problem) today. The measuring method adopted for thermal conductivity of a sample fluid is by acquiring data in transient time/temperature history far earlier than the stage in which convection occurs in the sample chamber. An IHCP for simultaneously estimating spatially varying thermal conductivity and heat capacity of a flash method was developed by Flach and Ozisik (1989). Many papers and books have been written on IHCP. Beck et al. (1996) summarized a short history and mathematical description of IHCP, and compared their experiment results. Such technology opened the way for non-destruction inspection of a harvest at farms.

Typical topics about the diagnosis of materials or the processes are as listed above, but there are few reports of an automatic control system for food processing. The problem making it difficult to control the heat-processing operation of food is as follows.

(1) Raw materials are organic in origin, which makes it hard to specify their thermal properties and therefore to specify the transfer function.

(2) Because the food is soft even if it is a solid food, the position of the sensor is easily upset. Even a slight force on the lead wire of the sensor will move the sensor. Thus, the position of the sensor is actually obscure.

(3) The responses of temperature in continuous media resemble a "dead time" component, making use of a feedback technique impossible.

In the following chapters, the basis of inverse analysis, and two topics presupposing that it incorporates a "future estimate technology" into food processing facilities will be described. The first topic is a system which has an appropriate retort control, estimating the future of the central temperature of the food body using the measurement value by a temperature sensor installed in an obscure position of the food body of which the thermal properties are unclear. The second topic is on the way of appropriately controlling the opening degree of an expansion valve of the freezing system in the food, of which thermal properties are strongly depended on temperature.

\section{The calculus of variations and the integral equations}

This chapter explains the simplest example of the inverse problem and the difference in two kinds of approach will be contrasted. The first approach is exercises the calculus of variations which the recent paper on IHCP often uses. The second approach is an answer method using the integral equation. The definition of the narrow sense or of the traditional about the inverse problem is an integral equation.

Consider a mass, $m$ slipping without friction from a higher point, $P(x=u, Y=v)$ to the lower origin, $O(x=0$, $y=0$ ) along a sliding guide that is in a vertical plane with the horizontal axis, $x$ and the vertical axis, $y$. The curve of the guide will be written by the following formula.

$$
y=y(x) \text { or } x=\phi(y)
$$

$$
y^{\prime} \equiv \frac{d y}{d x} \quad \text { or } \quad \frac{d x}{d t}=\frac{d \phi}{d y} \frac{d y}{d t}
$$

Assuming the mass starts from rest at a point, $P(u, v)$, and it reaches a position, $Q(x, y)$ with a velocity, $w$ by the time, $t$. The kinetic energy, $m w^{2} / 2$ is equal to the potential energy, $m g(v-y)$, so the following formula can be held, where $g$ is the acceleration constant due to gravity.

$$
\begin{gathered}
w^{2}=\left(\frac{d x}{d t}\right)^{2}+\left(\frac{d y}{d t}\right)^{2}=\left\{1+\left(\frac{d y}{d x}\right)^{2}\right\}\left(\frac{d x}{d t}\right)^{2} \\
=2 g(v-y) \\
t=\frac{1}{\sqrt{2 g}} \int_{0}^{u} \frac{\sqrt{1+(d y / d x)^{2}}}{\sqrt{v-y}} d x \equiv \frac{1}{\sqrt{2 g}} I(y):=\min
\end{gathered}
$$

$$
t=\frac{1}{\sqrt{2 g}} \int_{0}^{v} \frac{\sqrt{1+(d \phi / d y)^{2}}}{\sqrt{v-y}} d y \equiv \frac{1}{\sqrt{2 g}} f(v):=\text { given }
$$

Brachistochrone problem The function, $y(x)$ of an independent variable, $x$ is called the "argument function," and the function, $I(y)$ of the argument function, $y(x)$ is called the "functional." From Eq. (2.3),

$$
I=\int_{0}^{u} \frac{\sqrt{1+y^{\prime 2}}}{\sqrt{v-y}} d x \equiv \int_{0}^{u} F\left(x, y, y^{\prime}\right) d x
$$

To decide simultaneously the form and value of an argument function, $y(x)$ which gives the functional, $I(y)$ to be minimized is called the "calculus of variations".

An optional function, $\eta(x)$, which becomes zero at the both limits of integration, is introduced here.

$$
\eta(u)=\eta(0)=0
$$

A new function, $Y(x)$ namely a "comparison function" with a small parameter, $\alpha$ is introduced.

$$
\begin{aligned}
Y(x) & \equiv y(x)+\alpha \eta(x) \equiv y+\delta y \\
\therefore \quad I(Y)-I(y) & \\
& =\int_{0}^{u}\left\{F\left(x, y+\alpha \eta, y^{\prime}+\alpha \eta^{\prime}\right)-F\left(x, y, y^{\prime}\right)\right\} d x \\
& =\alpha \int_{0}^{u}\left(\frac{\partial F}{\partial y} \eta+\frac{\partial F}{\partial y^{\prime}} \eta^{\prime}\right) d x+\frac{\alpha^{2}}{2 !} \int_{0}^{u}\{\ldots\} d x
\end{aligned}
$$

The first term of the right-hand side is called the "first variational", $\delta I$.

$$
\delta I \equiv \alpha \int_{0}^{u}\left(\frac{\partial F}{\partial y} \eta+\frac{\partial F}{\partial y^{\prime}} \eta^{\prime}\right) d x
$$

The functional, $I(Y)$ will be shifted from the stationary value, because $y(x)$ gives it a stationary. Therefore, the following formula is concluded, when parameter $\alpha$ is 0 . 


$$
\begin{aligned}
\left.\frac{d I(Y)}{d \alpha}\right|_{\alpha=0} & =\left.\int_{0}^{u}\left(\frac{\partial F}{\partial Y} \frac{\partial Y}{\partial \alpha}+\frac{\partial F}{\partial Y^{\prime}} \frac{\partial Y^{\prime}}{\partial \alpha}\right) d x\right|_{\alpha=0} \\
& =\left.\int_{0}^{u}\left(\frac{\partial F}{\partial Y} \eta+\frac{\partial F}{\partial Y^{\prime}} \eta^{\prime}\right) d x\right|_{\alpha=0} \\
& =\int_{0}^{u}\left(\frac{\partial F}{\partial y} \eta+\frac{\partial F}{\partial y^{\prime}} \eta^{\prime}\right) d x=0
\end{aligned}
$$

Integrating by parts, it becomes

$$
\left.\frac{d I(Y)}{d \alpha}\right|_{\alpha=0}=\left.\frac{\partial F}{\partial y^{\prime}} \eta\right|_{0} ^{u}+\int_{0}^{u} \eta\left\{\frac{\partial F}{\partial y}-\frac{d}{d x}\left(\frac{\partial F}{\partial y^{\prime}}\right)\right\} d x
$$

Now the first term of the right-hand side is identical to zero because of the condition of Eq. (2.6). Therefore, the second term becomes

$$
\frac{\partial F}{\partial y}-\frac{d}{d x}\left(\frac{\partial F}{\partial y^{\prime}}\right)=0
$$

This relation is called the "Euler-Lagrange equation". The following differentiation can be held, if the independent variable, $x$ does not appear in the function, $F$ explicitly.

$$
\begin{gathered}
\frac{d}{d x}\left(F-y^{\prime} \frac{\partial F}{\partial y^{\prime}}\right)=y^{\prime}\left(\frac{\partial F}{\partial y}-y^{\prime} \frac{\partial^{2} F}{\partial y^{\prime} \partial y}-y^{\prime \prime} \frac{\partial^{2} F}{\partial y^{\prime 2}}\right) \\
=y^{\prime}\left\{\frac{\partial F}{\partial y}-\frac{d}{d x}\left(\frac{\partial F}{\partial y^{\prime}}\right)\right\}=0
\end{gathered}
$$

Accordingly, $F-y^{\prime} \frac{\partial F}{\partial y^{\prime}}=$ const.

$$
\text { or } \quad \frac{\sqrt{1+y^{\prime 2}}}{\sqrt{v-y}}-\frac{y^{\prime 2}}{\sqrt{v-y} \sqrt{1+y^{\prime 2}}}=\frac{1}{\sqrt{C_{1}}}
$$

Multiplying both sides by $\sqrt{1+y^{\prime 2}}$ gives

$$
\left(\frac{d y}{d x}\right)^{2}=\frac{C_{1}}{v-y}-1=\frac{C_{1}-v+y}{v-y}
$$

Using the parameter, $\vartheta$, and assuming

$$
y-v=\frac{C_{1}}{2}(\cos \vartheta-1)
$$

Then

$$
x-u=\frac{C_{1}}{2}(\vartheta-\sin \vartheta)
$$

Like this example, the variational principal is a natural extension of differential calculus. Lesnic et al. (1998) and Park et al. (1999) are using the variational principal for IHCP.

Tautochrone problem The new function $\Psi(y)$ is defined as

$$
\Psi(y) \equiv \sqrt{1+(d \phi / d y)^{2}}
$$

The integral equation (2.4) can be written as

$$
\int_{0}^{v} \frac{\Psi(y)}{\sqrt{v-y}} d y \equiv \int_{0}^{v} K(v, y) \Psi(y) d y=f(v)
$$

Generally, the term $K(v, y)$ is called the "kernel of integral". Dividing both sides by $\sqrt{\xi-v}$, and integrating between 0 and $\xi$ with respect to $v$, interchanging the order of integration yields

$$
\begin{aligned}
\int_{0}^{\xi} \frac{f(v) d v}{\sqrt{\xi-v}} & =\int_{0}^{\xi}\left\{\int_{0}^{v} \frac{\Psi(y) d y}{\sqrt{v-y}}\right\} \frac{d v}{\sqrt{\xi-v}} \\
& =\int_{0}^{\xi}\left\{\int_{y}^{\xi} \frac{d v}{\sqrt{v-y} \sqrt{\xi-v}}\right\} \Psi(y) d y
\end{aligned}
$$

Here

$$
\int_{y}^{\xi} \frac{d v}{\sqrt{v-y} \sqrt{\xi-v}}=\pi
$$

The "tautochrone" is a problem which decides a curve, that always makes constant time in which the mass reaches the terminal wherever it leaves, $f(v)=$ const. $\equiv C$,

$$
\Psi(y)=\frac{1}{\pi} \frac{d}{d y} \int_{0}^{\xi} \frac{C}{\sqrt{\xi-v}} d v \frac{C}{\pi \sqrt{y}}
$$

Substituting in Eq. (2.12),

$$
\frac{d x}{d y}=\sqrt{\frac{C^{2}}{\pi^{2} y}-1}
$$

The solution is as follows with the parameter, $\vartheta$.

$$
\begin{aligned}
& x=\frac{C^{2}}{\pi^{2}}(1-\cos \vartheta) \\
& y=\int_{0}^{\theta} \sqrt{\frac{1+\cos \vartheta}{1-\cos \vartheta}} \frac{C^{2}}{\pi^{2}} \sin \vartheta d \vartheta=\frac{C^{2}}{\pi^{2}}(\vartheta+\sin \vartheta)
\end{aligned}
$$

The typical IHCP is the type to solve an integral equation.

\section{Inspection of the model}

If the model is not expressing an actual phenomenon correctly, the result of the inverse analysis will be upset to the extent that it does not have a meaning physically. Most of the reports point out this empirical fact.

Sterilization effect In this text, the following four assumptions are introduced. The density of microorganism cells per unit volume is denoted as $N\left(\mathrm{~m}^{-3}\right)$.

(1) The reaction in the heat sterilization process can be assumed as the "first order". The slight microorganism live cells density, $d N$, which decreases during the slight time, $d t$ (s) of the heating, is proportional to density, $N$ at a position in the foods. Survival ratio, $\omega$ is defined as

$$
\omega \equiv N / N_{0},
$$

where $N_{0}$ is the initial density of a microorganism at $t=0$

$$
\frac{d \omega}{d t}=-k(T) \omega
$$


The time that the ratio, $\omega$ becomes $1 / 10$ is " $D$-value" (decimal reduction time).

$$
D(T) \equiv \ln (10) / k(T)
$$

where $k(T)$ is a function of temperature, $T(\mathrm{~K})$,

$$
\frac{d\{\ln (k)\}}{d T}=\frac{E}{\mathfrak{R} T^{2}}
$$

where $\Re\left(\mathrm{J} \mathrm{K}^{-1} \mathrm{~mol}^{-1}\right)$ is the gas constant, and $E\left(\mathrm{~J} \mathrm{~mol}^{-1}\right)$ is the apparent activation energy. Integrating between the reference temperature, $T_{\mathrm{R}} 394.26 \mathrm{~K}=250^{\circ} \mathrm{F}$, and $T$

$$
\ln \left(\frac{k\left(T_{\mathrm{R}}\right)}{k(T)}\right)=\frac{E}{\mathfrak{R} T}-\frac{E}{\mathfrak{R} T_{\mathrm{R}}}
$$

During cooking, the similar tendency is presented on supervening reactions to be avoided e.g. the decrease of nutritional concentration, the hardness and so on. In the botulinus bacillus; $k\left(T_{\mathrm{R}}\right) \approx 0.057 \mathrm{~s}^{-1}, E \approx 300 \mathrm{~kJ} \mathrm{~mol}^{-1}$, and in thiamine; $k\left(T_{\mathrm{R}}\right) \approx 0.009 \mathrm{~s}^{-1}, E \approx 115 \mathrm{~kJ} \mathrm{~mol}^{-1}$. Under identical sterilization effects, the processing is higher for the remaining nutritional percentage in a high temperature short time (HTS) than in a low temperature long time.

(2) Liner algebra can be usually adapted on this reaction, regardless of the temperature history. In other words, the sterilization effect when heated for 2 minutes at $120^{\circ} \mathrm{C}$ after it had been heated for 1 minute at $100^{\circ} \mathrm{C}$ is equal to the effect of heating for 1 minute at $100^{\circ} \mathrm{C}$ after heating for 2 minutes at $120^{\circ} \mathrm{C}$. That this is a mistake has been understood from Pasteur's times, but here, for simplification of the computation, we adopt this supposition.

(3) The local temperature, $T$ and the velocity components, $(u, v, w)$ with respect to the direction on the coordinate, $(x, y, z)$ in the liquid foods can be found using thermofluid dynamics. Accordingly here, they can be assumed as given functions. The conservation law of microorganism cells is due to the following formula. Ignore the self diffusion effects such as drifting of the microorganism.

$$
\frac{\partial \omega}{\partial t}+u \frac{\partial \omega}{\partial x}+v \frac{\partial \omega}{\partial y}+w \frac{\partial \omega}{\partial z}=-k(T) \omega
$$

A goal value is made $\omega \approx 10^{-12}$ in the commercial sterilization.

(4) The following formula can be assumed within a narrow range of temperature

$$
\log _{10}\left(\frac{D(T)}{D\left(T_{\mathrm{R}}\right)}\right)=\frac{E\left(T_{\mathrm{R}}-T\right)}{\Re T T_{\mathrm{R}} \ln (10)} \rightleftharpoons \frac{E\left(T_{\mathrm{R}}-T\right)}{\Re T_{\mathrm{R}}^{2} \ln (10)} \frac{T_{\mathrm{R}}-T}{Z}
$$

where the constant, $Z\left({ }^{\circ} \mathrm{C}\right)$ is called $Z$-value. The first assumption becomes the formula below.

$$
D\left(T_{\mathrm{R}}\right) \log _{10}\left(\frac{N_{0}}{N}\right)=10^{\frac{T-T_{\mathrm{R}}}{Z}} \cdot t
$$

When planning a sterilization goal, $\omega$ which has safe practicality, it is possible to assume that the left-hand side of Eq. (3.7) is the time equivalent to the goal value. This will be written with $F_{\mathrm{R}}$.

$$
F_{\mathrm{R}} \equiv-D\left(T_{\mathrm{R}}\right) \log _{10} \omega
$$

The right-hand side of Eq. (3.7), is called the " $F$ value," "sterilization effect," "lethality," or "sterility" as a practical effect in the operation time. From the second assumption of the liner algebra, it is reasonable to integrate over all the processes of the heating and cooling.

$$
F=\int \exp \left\{\frac{T-T_{\mathrm{R}}}{Z}\right\} \ln (10) d t
$$

Governing equation of heat conduction For an easy explanation here, we assume the thin type retort pouch or the flat board type of large canned foods which has attracted the attention of business of recently because of the excellent convenience of stacking storage, heat transfer performance and so on. The arithmetic and results are shown in a representative instance in an infinite slab of solid food body with thickness $2 D(\mathrm{~m})$. The governing differential equation of heat conduction can be reduced as one dimension,

$$
\rho c_{\mathrm{p}} \frac{\partial T}{\partial t}=\frac{\partial}{\partial x}\left(\lambda \frac{\partial T}{\partial x}\right)
$$

Appropriate boundary conditions are

$$
\left.h\left(T-T_{\mathrm{H}}\right)\right|_{\mathrm{x}= \pm \mathrm{D}}=-\left.\lambda \frac{\partial T}{\partial T}\right|_{\mathrm{x}= \pm \mathrm{D}}
$$

where $T_{\mathrm{H}}(\mathrm{K})$ is temperature of the heating medium, $\rho(\mathrm{kg}$ $\left.\mathrm{m}^{-3}\right)$ is the food density, $c_{\mathrm{p}}\left(\mathrm{kJ} \mathrm{kg}^{-1} \mathrm{~K}^{-1}\right)$ is the heat capacity of food, $\lambda\left(\mathrm{W} \mathrm{m}^{-1} \mathrm{~K}^{-1}\right)$ is the thermal conductance of the food and $h\left(\mathrm{~W} \mathrm{~m}^{-2} \mathrm{~K}^{-1}\right)$ is the heat transfer coefficient on the food body surface. Then, three new parameters are introduced,

$$
\begin{aligned}
& \xi \equiv \frac{x}{D}: \text { Non-dimensional position, } \\
& \tau \equiv \frac{\rho c_{\mathrm{p}} D^{2}}{\lambda}: \text { Time constant, } \\
& B i \equiv \frac{h D}{\lambda}: \text { Biot number. }
\end{aligned}
$$

The initial temperature of food is uniformly constant, $T_{0}$. The temperature of heating medium, $T_{\mathrm{H}}$ in the retort changes continuously but this can be assumed to be a lot of change of the stepwise function. At the time step, $t_{\mathrm{k}}(s)$, the temperature of the heating medium changes to $T_{\mathrm{k}}$. Then, a new time variable, $\eta_{\mathrm{k}}$ is introduced.

$$
\begin{aligned}
& 1 \mathrm{st}, \eta_{1}=(t-0) / \tau, T_{\mathrm{H}}=T_{1}, \\
& \text { 2nd, } \eta_{2}=\left(t-t_{2}\right) / \tau, T_{\mathrm{H}}=T_{2}, \\
& \ldots, \ldots, \ldots, \\
& k \text { th, } \eta_{\mathrm{k}}=\left(t-t_{\mathrm{k}}\right) / \tau, T_{\mathrm{H}}=T_{\mathrm{k}},
\end{aligned}
$$


The non-dimensional temperature, $\theta$ can be defined as

$$
\theta_{\mathrm{k}} \equiv \frac{T-T_{\mathrm{k}}}{T_{\mathrm{k}-1}-T_{\mathrm{k}}}
$$

Substituting these non-dimensional parameters into Eqs. (3.11) and (3.12) give

$$
\begin{gathered}
\frac{\partial \theta_{\mathrm{k}}}{\partial \eta_{\mathrm{k}}}=\frac{\partial^{2} \theta_{\mathrm{k}}}{\partial \xi^{2}} \\
\pm\left.\frac{\partial \theta_{\mathrm{k}}}{\partial \xi}\right|_{\xi= \pm 1}=-\left.B i \theta_{\mathrm{k}}\right|_{\xi= \pm 1}
\end{gathered}
$$

The analytical solutions are

$$
\begin{aligned}
& \theta_{1}=\sum_{n=1}^{\infty} A_{\mathrm{n}} \exp \left\{-Q_{\mathrm{n}}^{2} \eta_{1}\right\} \cos \left(Q_{\mathrm{n}} \xi\right) \\
& \theta_{2}=\sum_{n=1}^{\infty}\left\langle C_{\mathrm{n}}\right\rangle_{2} \exp \left\{-Q_{\mathrm{n}}^{2} \eta_{2}\right\} \cos \left(Q_{\mathrm{n}} \xi\right) \\
& \ldots, \ldots, \\
& \theta_{\mathrm{k}}=\sum_{n=1}^{\infty}\left\langle C_{\mathrm{n}}\right\rangle_{\mathrm{k}} \exp \left\{-Q_{\mathrm{n}}^{2} \eta_{\mathrm{k}}\right\} \cos \left(Q_{\mathrm{n}} \xi\right)
\end{aligned}
$$

Where eigen value, $Q_{\mathrm{n}}$ is an $n$th root of the following equation,

$$
Q_{\mathrm{n}} \tan Q_{\mathrm{n}}=B_{\mathrm{i}}
$$

the Fourier coefficients, $A_{\mathrm{n}}$ due to the first heating phase, and $\left\langle C_{\mathrm{n}}\right\rangle_{\mathrm{k}}$, due to the $k$-th processes are

$$
\begin{gathered}
A_{\mathrm{n}} \equiv 2(-1)^{\mathrm{n}} Q_{\mathrm{n}} \\
\left\langle C_{\mathrm{n}}\right\rangle_{2}=\frac{T_{0}-T_{1}}{T_{1}-T_{2}} A_{\mathrm{n}} \exp \left\{-Q_{\mathrm{n}}^{2} \frac{t_{2}}{\tau}\right\}+A_{\mathrm{n}} \\
\ldots \\
\left\langle C_{\mathrm{n}}\right\rangle_{\mathrm{k}}=\left\langle C_{\mathrm{n}}\right\rangle_{\mathrm{k}-1}\left[1+\frac{T_{\mathrm{k}-2}-T_{\mathrm{k}-1}}{T_{\mathrm{k}-1}-T_{\mathrm{k}}} \exp \left\{-P_{\mathrm{n}}^{2} \frac{t_{\mathrm{k}}-t_{\mathrm{k}-1}}{\tau}\right\}\right]
\end{gathered}
$$

As can be understood by the above analysis, only two parameters $\tau$ and $B_{i}$ are dominating this phenomenon. Accordingly, they can be estimated if they are equal to or more than two observation values. For details of the way of inverse analyzing for this purpose, there is a small paragraph in chapter 4.

Analytical solution of food freezing Most foods include about 80 percent water. In the freezing process, a two-phase region disperses pure ice crystals within the aqueous solution. The concentration of solute in the aqueous phase surrounding an ice crystal increases as temperature falls. New crystals nucleate in lean concentrate a short distance from the original ice crystal. This results in considerable change in thermal properties such as the effective thermal conductivity, $\lambda_{\mathrm{ef}}\left(\mathrm{J} \mathrm{m}^{-1} \mathrm{~K}^{-1}\right)$, the apparent heat capacity, $C_{\mathrm{p}}\left(\mathrm{J} \mathrm{m}^{-3} \mathrm{~K}^{-1}\right)$, and the apparent latent heat of solidification, $\rho L\left(\mathrm{~J} \mathrm{~m}^{-3}\right)$. The governing equation for conservation of heat flux, $q\left(\mathrm{~W} \mathrm{~m}^{-2}\right)$ with dispersed latent heat source, $\rho L\left(\mathrm{~J} \mathrm{~m}^{-3}\right)$ for solidification in one dimension can be written as

$$
\frac{\partial C p T}{\partial t}+\rho L \frac{\partial F_{\mathrm{f}}}{\partial t}+\frac{\partial q}{\partial x}=0
$$

where $F_{\mathrm{f}}$ is the volumetric liquid fraction which is defined as the volume of unfrozen solution divided by the total volume of the food. Since precise data on thermal properties is required in order to develop the theory, Mihori et al. $(1990,1992,1994 a, b)$ used an aqueous solution of sodium chloride (salt) as a model food. The phase equilibrium concentration, $C$ in the aqueous salt solution was successfully represented as

$$
C=\left(\frac{T}{K}\right)^{\mathrm{n}}
$$

where $n=0.746$ and $K=-143.43^{\circ} \mathrm{C}$. The extent of freezing, $R$ is defined by the mass of ice divided by the total mass,

$$
R=1-\frac{C_{0}}{C}=1-\left(\frac{T_{\mathrm{f}}}{T}\right)^{\mathrm{n}}
$$

where $C_{0}$ is the initial concentration and $T_{\mathrm{f}}$ is the initial freezing temperature. The $F_{\mathrm{f}}$ may be described as a function of temperature as

$$
F_{\mathrm{f}}=\frac{\rho_{\text {ice }}}{\rho_{\text {ice }}+R \rho_{\text {ice }}}=\frac{\rho_{\text {ice }}}{\rho_{\text {ice }}+\rho_{\mathrm{m}}\left\{1-\left(T_{\mathrm{f}} / T\right)^{\mathrm{n}}\right\}}
$$

where $\rho$ is density, and the subscripts "ice" and " $m$ " refer to pure water ice and unfrozen solution, respectively. The effective heat capacity per unit volume, $C_{\mathrm{p}}$ in the two-phase region can be written as

$$
C_{\mathrm{p}}=\left(1-F_{\mathrm{f}}\right) \rho_{\mathrm{ice}} C_{\mathrm{p} \text { ice }}+F_{\mathrm{f}} \rho_{\mathrm{m}} C_{\mathrm{pm}}
$$

Considering the temperature-variable nature of density, heat capacity, and liquid fraction in the two-phase region, the first term in Eq. (3.22) can be written as

$$
\begin{aligned}
& \frac{\partial C p T}{\partial t}=\left(\rho_{\mathrm{m}} C p_{\mathrm{m}}-\rho_{\text {ice }} C p_{\text {ice }}\right) T \frac{d F_{\mathrm{f}}}{d T} \frac{\partial T}{\partial t} \\
& +\left(1-F_{\mathrm{f}}\right)\left\{\rho_{\text {ice }} C p_{\text {ice }}+T\left(\frac{d \rho_{\text {ice }}}{d T} C p_{\text {ice }}+\rho_{\text {ice }} \frac{d C p_{\text {ice }}}{d T}\right)\right\} \frac{\partial T}{\partial t} \\
& +F_{\mathrm{f}}\left\{\rho_{\mathrm{m}} C p_{\mathrm{m}}+T\left(\frac{d \rho_{\mathrm{m}}}{d T} C p_{\mathrm{m}}+\rho_{\mathrm{m}} \frac{d C p_{\mathrm{m}}}{d T}\right)\right\} \frac{\partial T}{\partial t} \\
& =\Omega \frac{\partial T}{\partial t}
\end{aligned}
$$

The second term of Eq. (3.22) may be written as

$$
\rho_{\mathrm{f}} L \frac{\partial F_{\mathrm{f}}}{\partial t}=\rho_{\mathrm{f}} L \frac{d F_{\mathrm{f}}}{d T} \frac{\partial T}{\partial t} \equiv \Phi \frac{\partial T}{\partial t}
$$

The sum of $\Phi$ and $\Omega$, each of which is defined in Eqs. (3.27) and (3.28) respectively, can be expressed as a function of temperature.

The solid line in Fig. 1 shows $\Phi+\Omega$ calculated using Eqs. (3.27) and (3.28). This solid line can be approximated by the following equation (a dashed line in Fig. 1), 


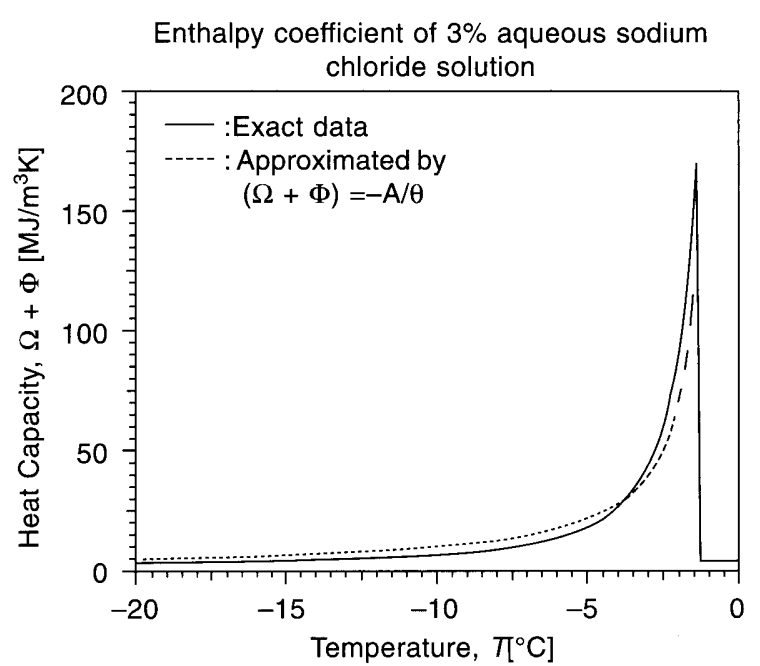

Fig. 1. The temperature tendency of the enthalpy coefficient, $\Phi+\Omega$ The solid line calculated by Eqs. (3.25) and (3.26)

The dashed line shows approximation Eq. (3.29).

$$
\begin{gathered}
\Phi+\Omega=-\frac{A}{\theta} \\
\theta=T+T_{\mathrm{c}}
\end{gathered}
$$

where $T_{\mathrm{c}}$ is a constant.

The heat flux in the third term of Eq. (3.22) can be written as

$$
q=-\lambda_{\text {ef }} \frac{\partial T}{\partial x}
$$

The $\lambda_{\text {ef }}$ can be expressed as shown by the solid line in Fig. 2. In the food freezing process the parallel model fit is well known.

$$
\lambda_{\mathrm{ef}}=\left(1-F_{\mathrm{f}}\right) \lambda_{\text {ice }}+F_{\mathrm{f}} \lambda_{\mathrm{m}}
$$

Since the range of $\lambda_{\mathrm{ef}}$ is much less than that of $\Phi+\Omega$, $\lambda_{\text {ef }}$ may be approximated by a linear function (chain line in Fig. 2) as

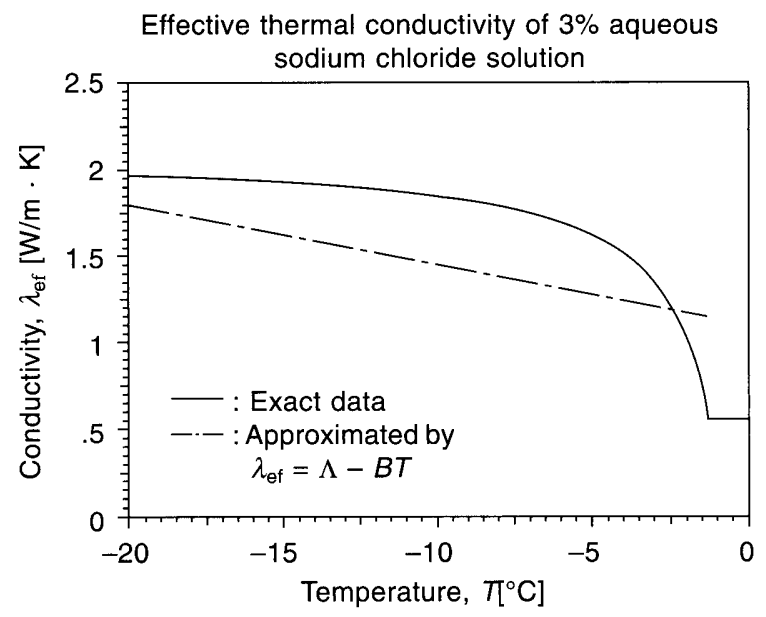

Fig. 2. The temperature tendency of the effective thermal conductivity, $\lambda_{\text {ef }}$. The solid line shows Eq. (3.32). The chain line shows approximation Eq. (3.33).

$$
\lambda_{\text {ef }}=\Lambda-B T,
$$

where $\Lambda$ and $B$ are constants.

Substituting Eqs. (3.24)-(3.33) into Eq. (3.22) yields

$$
-\frac{\partial \theta}{\partial t}=\theta \frac{\partial}{\partial x}\left\{(\beta-\Gamma \theta) \frac{\partial \theta}{\partial x}\right\}
$$

Where

$$
\beta \equiv \frac{\Lambda-B T}{A}, \Gamma \equiv \frac{B}{A}
$$

Converting the variables as

$$
\varsigma \equiv \pm x \sqrt{2 \Gamma t}
$$

The "freezing equation (3.34)" yields an ordinary differential equation as

$$
\theta\left\{\left(\theta-\frac{\beta}{\Gamma}\right) \frac{d^{2} \theta}{d \varsigma^{2}}+\left(\frac{d \theta}{d \varsigma}\right)^{2}\right\}-\varsigma \frac{d \theta}{d \zeta}=0
$$

Since the equation $\theta=\varsigma$ satisfies Eq. (3.37), it indicates the existence of a linear temperature profile in the twophase region. The heat flux, $q_{\mathrm{s}}$ from the surface temperature, $T_{\mathrm{S}}$ of the food body to the ambient coolant temperature, $T_{\mathrm{A}}$ may be equated to the heat conduction flux inside the body. The expression for $q_{\mathrm{s}}$ may be written using a heat transfer coefficient, $h$, and the position, $x_{\mathrm{f}}(\mathrm{m})$, at which the linear temperature profile intersects the freezing temperature, $T_{\mathrm{f}}$,

$$
q_{\mathrm{s}}=h\left(T_{\mathrm{S}}-T_{\mathrm{A}}\right)=\lambda_{\text {ef }} \frac{T_{\mathrm{f}}-T_{\mathrm{S}}}{x_{\mathrm{f}}-D}
$$

The solution is

$$
T_{\mathrm{S}}=\frac{T_{\mathrm{f}}+\left(1+\xi_{\mathrm{f}}\right) B i T_{\mathrm{A}}}{1+\left(1-\xi_{\mathrm{f}}\right) B i}
$$

where $\quad B i \equiv \frac{D h}{\lambda_{\text {ef }}}, \xi_{\mathrm{f}} \equiv \frac{x_{\mathrm{f}}}{D}, D=$ half thickness.

Now, $q_{\mathrm{S}}$ is equated to the rate of enthalpy change inside the body,

$$
\begin{aligned}
& q_{\mathrm{S}}=\frac{d}{d t} \int_{D}^{x_{\mathrm{f}}} \int_{T_{\mathrm{S}}}^{T_{\mathrm{f}}}(\Phi+\Omega) d T d x \\
& =\frac{d}{d t}\left\{\left(x_{f}-D\right) \int_{T_{\mathrm{S}}}^{T_{\mathrm{f}}}(\Phi+\Omega) d T\right\}
\end{aligned}
$$

where $Q_{\mathrm{F}} \equiv \int(\Phi+\Omega) d T$ may be independent of the lower limit of integration, because the value $(\Phi+\Omega)$ vanishes at the lower temperatures. From Eqs. (3.38)-(3.41),

$$
\frac{d t}{\tau_{\mathrm{f}}}=\frac{T_{\mathrm{f}}-T_{\mathrm{A}}}{T_{\mathrm{f}}-T_{\mathrm{S}}}\left(1-\xi_{\mathrm{f}}\right) d\left(1-\xi_{\mathrm{f}}\right)
$$

The solution of which is 


$$
\begin{aligned}
& \xi_{\mathrm{f}}=1+\frac{1}{B i}-\sqrt{\frac{1}{B i^{2}}+\frac{2 t}{\tau_{\mathrm{f}}}} \\
& T=T_{\mathrm{A}}+\left(T_{\mathrm{f}}-T_{\mathrm{A}}\right) \frac{1+\left(1-\xi_{\mathrm{f}}\right) B i}{\sqrt{1+2 B i^{2} t / \tau_{\mathrm{f}}}}
\end{aligned}
$$

where $\tau_{\mathrm{f}} \equiv \frac{Q D^{2}}{\lambda_{\text {ef }}\left(T_{\mathrm{f}}-T_{\mathrm{A}}\right)}=$ time const.

After the freezing front reaches the center of the food body, the heat conduction equation with constant coefficient applies. Agreement of the above model was verified in detail by Sanz et al. (1999).

Generalized model for food freezing An analytical solution was found for the one-dimensional problem from the approximation of Eqs. (3.29) and (3.33). Unfortunately, the analytical solution for a two- or three-dimensional problem has not yet been discovered, even using these approximations. In this case, the following heat conduction equation for food during freezing should be solved through numerical calculation.

$$
\psi(T) \frac{\partial T}{\partial t}=\frac{\partial}{\partial x}\left\{\lambda(T)\left(\frac{\partial T}{\partial x}+\frac{\partial T}{\partial y}+\frac{\partial T}{\partial z}\right)\right\}
$$

Thermal properties of food change considerably during freezing and may be described as a function of temperature such as

$$
\begin{aligned}
& \lambda(T)=P_{1}+P_{2} \cdot\left(T_{\mathrm{F}}-T\right)^{\mathrm{P}_{3}} \\
& \psi(T)=P_{4} \cdot\left(T_{\mathrm{F}}-T\right)^{\mathrm{P}_{5}}
\end{aligned}
$$

where $P_{\mathrm{j}}$ are the coefficients for the thermal properties that should be estimated by inverse analysis.

\section{An example of inverse analysis systems}

In this chapter, the manner of parameter identification will be discussed.

Parameter identification from temperature records The procedure to estimate the parameter contained in the solution of the governing heat conduction equation using a set of data of the time/temperature record will be discussed here. The solution, $T_{\mathrm{i}}(\mathrm{K})$ of the heat conduction equation at time, $S_{\mathrm{i}}(\mathrm{s})$, may be written as

$$
T_{\mathrm{i}}(\mathbf{P}) \equiv T\left(S_{\mathrm{i}}, P_{1}, P_{2}, \ldots, P_{\mathrm{j}}, \ldots P_{\mathrm{L}}\right)
$$

where $S_{\mathrm{i}}$ is the $i$-th observation time, and $P_{\mathrm{j}}$ is the $j$-th unknown parameter, $L$ is the number of unknown parameters, and $\mathbf{P}$ denotes a row vector, $\left(P_{1}, P_{2}, \ldots, P_{\mathrm{j}}\right.$, $\left.\ldots, P_{\mathrm{L}}\right)$.

When $Y_{\mathrm{i}}$ denotes the observed temperature at $S_{\mathrm{i}}$, the error, $E_{\mathrm{i}}$ may be defined as

$$
E_{\mathrm{i}} \equiv T_{\mathrm{i}}\left(\mathbf{P}_{\mathrm{T}}\right)-Y_{\mathrm{i}}, i=1,2, \ldots, n .
$$

Subscript " $T$ " indicates the true values, $\mathbf{P}_{\mathrm{T}} \equiv\left(P_{1 \mathrm{~T}}, P_{2 \mathrm{~T}}\right.$, $\left.\ldots, P_{\mathrm{jT}}, \ldots, P_{\mathrm{LT}}\right)$ denotes the true value of the parameters and $n$ denotes the number of observed data sets. Our goal is to estimate $\mathbf{P}_{\mathrm{T}}$ that is not known at this moment. If the observed temperature, $Y_{\mathrm{i}}$ is disturbed by a white noise, the following may hold at $P_{\mathrm{j}}=P_{\mathrm{jT}}$,

$$
\frac{\partial}{\partial P_{\mathrm{j}}} \sum_{i=1}^{n} E_{\mathrm{i}}^{2}=0
$$

Since $\mathrm{P}_{\mathrm{T}}$ is unknown, we cannot calculate $E_{\mathrm{i}}$. As an alternative to the true parameter, $\mathrm{P}_{\mathrm{T}}$, we might guess at the value, $\mathbf{P}_{y}$. Hence, the true temperature, $T_{\mathrm{i}}\left(\mathbf{P}_{\mathrm{T}}\right)$ may be described using a variance, $\delta T_{\mathrm{i}}(\mathbf{P})$ as

$$
T_{\mathrm{i}}\left(\mathrm{P}_{\mathrm{T}}\right)=T_{\mathrm{i}}\left(\mathrm{P}_{\mathrm{y}}\right)+\delta T_{\mathrm{i}}(\mathrm{P})
$$

while the relevant variance of $\mathrm{P}_{\mathrm{y}}$ may be denoted by $\delta P$,

$$
\mathrm{P}_{\mathrm{T}}=\mathrm{P}_{\mathrm{y}}+\delta \mathrm{P}
$$

Substituting Eq. (4.4) into Eq. (4.2), the observation error, $E_{\mathrm{i}}$ may be written as

$$
\begin{gathered}
E_{\mathrm{i}}=T_{\mathrm{i}}\left(\mathrm{P}_{\mathrm{y}}\right)+\delta T_{\mathrm{i}}(\mathrm{P})-Y_{\mathrm{i}} \\
E_{\mathrm{i}}=R_{\mathrm{i}}+\delta T_{\mathrm{i}}(\mathrm{P})
\end{gathered}
$$

where the residual, $R_{\mathrm{i}}$ is defined by

$$
R_{\mathrm{i}} \equiv T_{\mathrm{i}}\left(\mathrm{P}_{\mathrm{y}}\right)-Y_{\mathrm{i}}
$$

By use of the variance, we can calculate $E_{\mathrm{i}}$. Substituting Eq. (4.6) into Eq. (4.3), we have for $j=1,2, \ldots, L$,

$$
\frac{\partial}{\partial \mathrm{P}_{\mathrm{j}}} \sum_{i=1}^{n}\left\{T_{\mathrm{i}}\left(\mathrm{P}_{\mathrm{y}}\right)+\delta T_{\mathrm{i}}(\mathrm{P})-Y_{\mathrm{i}}\right\}^{2}=0
$$

where the term $\partial / \partial P_{\mathrm{j}}$ denotes a partial derivative with respect to $P_{\mathrm{j}}$ at $\mathrm{P}=\mathrm{P}_{\mathrm{y}}$. This can be rewritten as follows for $j=1,2, \ldots, L$.

$$
\begin{aligned}
& 2 \sum_{i=1}^{n}\left[\left\{T_{\mathrm{i}}\left(\mathrm{P}_{\mathrm{y}}\right)+\delta T_{\mathrm{i}}(\mathrm{P})-Y_{\mathrm{i}}\right\} \frac{\partial}{\partial P_{\mathrm{j}}}\left\{T_{\mathrm{i}}\left(\mathrm{P}_{\mathrm{y}}\right)+\delta T_{\mathrm{i}}(\mathrm{P})-Y_{\mathrm{i}}\right\}\right] \\
& \quad=0
\end{aligned}
$$

Since $Y_{\mathrm{i}}$ is constant, Eq. (4.10) may be reduced to

$$
2 \sum_{i=1}^{n}\left[\left\{R_{\mathrm{i}}+\delta T_{\mathrm{i}}(\mathrm{P})\right\} \frac{\partial}{\partial P_{\mathrm{j}}}\left\{T_{\mathrm{i}}\left(\mathrm{P}_{\mathrm{y}}\right)+\delta T_{\mathrm{i}}(\mathrm{P})\right\}\right]=0
$$

Now, two typical situations can be considered:

\section{Case A:}

If the guessed at value $\mathrm{P}_{\mathrm{y}}$ is a good one, the variance $\delta T_{\mathrm{i}}(\mathrm{P})$ may be very small,

$$
\operatorname{Order}\left[T_{\mathrm{i}}\left(\mathrm{P}_{\mathrm{y}}\right)\right]>>\operatorname{Order}\left[\delta T_{\mathrm{i}}(\mathrm{P})\right]
$$

Then Eq. (4.11) yields

$$
\sum_{i=1}^{n}\left[\left\{R_{\mathrm{i}}+\delta T_{\mathrm{i}}(\mathrm{P})\right\} \frac{\partial T_{\mathrm{i}}\left(\mathrm{P}_{\mathrm{y}}\right)}{\partial P_{\mathrm{j}}}\right]=0
$$

On the other hand, the variance at $P=P_{\mathrm{y}}$ may be written as

$$
\delta T_{\mathrm{i}}(\mathrm{P})=\sum_{j=1}^{L}\left[\frac{\partial T_{\mathrm{i}}\left(\mathrm{P}_{\mathrm{y}}\right)}{\partial P_{\mathrm{j}}} \delta P_{\mathrm{j}}\right]
$$


Substituting Eq. (4.14) into Eq. (4.13), gives

$$
\sum_{i=1}^{n}\left[R_{\mathrm{i}} \frac{\partial T_{\mathrm{i}}\left(\mathrm{P}_{\mathrm{y}}\right)}{\partial P_{\mathrm{k}}}+\sum_{j=1}^{L}\left\{\frac{\partial T_{\mathrm{i}}\left(\mathrm{P}_{\mathrm{y}}\right)}{\partial P_{\mathrm{k}}} \frac{\partial T_{\mathrm{i}}\left(\mathrm{P}_{\mathrm{y}}\right)}{\partial P_{\mathrm{j}}} \delta P_{\mathrm{j}}\right\}\right]=0
$$

where $k=1,2, \ldots, L$.

This equation is well known as "Taylor's method" or the "steepest-descent method" for data fitting. As long as the assumption (4.12) holds, this method provides the fastest fitting tool. However, this does not work when the assumption (4.12) does not hold.

Case B:

If the guessed at value for $P_{\mathrm{y}}$ is not a good one, the order of variance $\delta T_{i}(\mathrm{P})$ may be equal to the order of $T_{\mathrm{i}}\left(\mathrm{P}_{\mathrm{y}}\right)$.

$$
\operatorname{Order}\left[T_{\mathrm{i}}\left(\mathrm{P}_{\mathrm{y}}\right)\right] \approx \operatorname{Order}\left[\delta T_{\mathrm{i}}(\mathrm{P})\right]
$$

In this case the last term in Eq. (4.11) cannot be discarded. Differentiating Eq. (4.14) with respect to $P_{\mathrm{j}}$,

$$
\frac{\partial \delta T_{\mathrm{i}}(\mathrm{P})}{\partial P_{\mathrm{j}}}=\sum_{j=1}^{L}\left\{\frac{\partial^{2} T_{\mathrm{i}}\left(\mathrm{P}_{\mathrm{y}}\right)}{\partial P_{\mathrm{j}}^{2}} \delta P_{\mathrm{j}}\right\}+\frac{\partial T_{\mathrm{i}}\left(\mathrm{P}_{\mathrm{y}}\right)}{\partial P_{\mathrm{j}}}
$$

substituting Eq. (4.17) into Eq. (4.11) gives

$$
\sum_{i=1}^{n}\left\{R_{\mathrm{i}}+\delta T_{\mathrm{i}}(\mathrm{P})\right\}\left\{\sum_{j=1}^{L}\left[\frac{\partial^{2} T_{\mathrm{i}}\left(\mathrm{P}_{\mathrm{y}}\right)}{\partial P_{\mathrm{j}}^{2}} \delta P_{\mathrm{j}}\right]+2 \frac{\partial T_{\mathrm{i}}\left(\mathrm{P}_{\mathrm{y}}\right)}{\partial P_{\mathrm{j}}}\right\}=0
$$

Equation (4.18) is a second order simultaneous equation of $\delta P_{\mathrm{j}}$, because $\delta T_{\mathrm{i}}(\mathrm{P})$ is a function of $\delta P_{\mathrm{j}}$. We cannot solve it as it is. Fortunately however, we can reach the goal by setting an equation:

$$
\left\{R_{\mathrm{i}}+\delta T_{\mathrm{i}}(\mathrm{P})\right\}=0
$$

the solution of which satisfies Eq. (4.11). Substituting Eq. (4.14) into (4.19).

$$
\begin{gathered}
-R_{\mathrm{i}} \approx \delta T_{\mathrm{i}}(\mathrm{P})=\sum_{j=1}^{L}\left\{\frac{\partial T_{\mathrm{i}}\left(\mathrm{P}_{\mathrm{y}}\right)}{\partial P_{\mathrm{j}}} \delta P_{\mathrm{j}}\right\} \\
\sum_{i=1}^{n} \sum_{j=1}^{L}\left\{\frac{\partial T_{\mathrm{i}}\left(\mathrm{P}_{\mathrm{y}}\right)}{\partial P_{\mathrm{j}}} \delta P_{\mathrm{j}}\right\} \approx-\sum_{i=1}^{n} R_{\mathrm{i}}
\end{gathered}
$$

Equations (4.15) and (4.20) are the $L$-th simultaneous equation. We first choose a commonplace value as a guess parameter values. The simultaneous equation is solved and the true parameters are estimated. Using these estimated values for new guess values, we repeat the same procedure. These routines are repeated successively until the revision quantities become very small. The iterating calculation using this procedure was found to be very stable and useful when the guessed at values are not near the true values, although it becomes very slow when the guessed at values approach the true values.

In The Second Half Stage of the search for the true parameters, $P_{\mathrm{T}}$, the guessed at values, $P_{\mathrm{y}}$ may have slight errors. Consequently one can take advantage of Taylor's method. For convenience in computer coding, introducing row vectors, $A$ and $B$, whose elements are

$$
\begin{gathered}
A_{\mathrm{j}} \equiv \sum_{i=1}^{n} \frac{\partial T_{\mathrm{i}}\left(P_{\mathrm{y}}\right)}{\partial P_{\mathrm{j}}} \\
B_{\mathrm{j}} \equiv \sum_{i=1}^{n} R_{\mathrm{i}} \frac{\partial T_{\mathrm{i}}\left(P_{\mathrm{y}}\right)}{\partial P_{\mathrm{j}}}
\end{gathered}
$$

A "Hessian matrix," $H$ may be defined as,

$$
H \equiv(A)^{t} A
$$

where (. . . $)^{\mathrm{t}}$ denotes a transposed (or column) vector. Using these notations, Taylor's equation (4.15) can be written as

$$
H \delta P=-(B)^{\mathrm{t}}
$$

where $\delta P$ is a vector.

In The First Half Stage of the search, one can take advantage of Eq. (4.20). Substituting Eq. (4.21) into (4.22), we have

$$
B_{\mathrm{j}}=R_{\mathrm{i}} A_{\mathrm{j}}
$$

and Eq. (4.20) may be written as

$$
A_{\mathrm{j}} A_{\mathrm{j}} \delta P_{\mathrm{j}} \approx-B_{\mathrm{j}}
$$

Equation (4.26) can be solved using the same scheme as used for solving Eq. (4.24); in the case of Eq. (4.26), however, the diagonal elements of Eq. (4.23) should be multiplied by a weighting factor, $\sigma$. The weighting factor, $\sigma$, described as follows, was found to work.

$$
\sigma \equiv 1+\frac{1}{n} \sum_{i=1}^{n} R_{\mathrm{i}}^{2}
$$

An on-line control system for heat sterilization During the early stages of the heating process, this system collects a series of time temperature data via two sensing probes which installed in the food body and heating medium in the retort. As described in section 4.1, the system analyzes inversely the data to estimate an inserted position, $\xi$ of sensor, as well as thermal parameters of the food such as Biot number, $B i$ and time constant, $\tau$. Equation (4.24) can be written as

$$
\begin{gathered}
\sum_{i=1}^{n}\left(R_{\mathrm{i}} \frac{\partial \theta}{\partial \tau}\right)+\sigma \sum_{i=1}^{n}\left(\frac{\partial \theta}{\partial \tau} \frac{\partial \theta}{\partial \tau}\right) \delta \tau \\
+\sum_{i=1}^{n}\left(\frac{\partial \theta}{\partial \tau} \frac{\partial \theta}{\partial B i}\right) \delta B i+\sum_{i=1}^{n}\left(\frac{\partial \theta}{\partial \tau} \frac{\partial \theta}{\partial \xi}\right) \delta \xi=0 \\
\sum_{i=1}^{n}\left(R_{\mathrm{i}} \frac{\partial \theta}{\partial B i}\right)+\sum_{i=1}^{n}\left(\frac{\partial \theta}{\partial B i} \frac{\partial \theta}{\partial \tau}\right) \delta \tau \\
+\sigma \sum_{i=1}^{n}\left(\frac{\partial \theta}{\partial B i} \frac{\partial \theta}{\partial B i}\right) \delta B i+\sum_{i=1}^{n}\left(\frac{\partial \theta}{\partial B i} \frac{\partial \theta}{\partial \xi}\right) \delta \xi=0 \\
\sum_{i=1}^{n}\left(R_{\mathrm{i}} \frac{\partial \theta}{\partial \xi}\right)+\sum_{i=1}^{n}\left(\frac{\partial \theta}{\partial \xi} \frac{\partial \theta}{\partial \tau}\right) \delta \tau \\
+\sum_{i=1}^{n}\left(\frac{\partial \theta}{\partial \xi} \frac{\partial \theta}{\partial B i}\right) \delta B i+\sigma \sum_{i=1}^{n}\left(\frac{\partial \theta}{\partial \xi} \frac{\partial \theta}{\partial \xi}\right) \delta \xi=0
\end{gathered}
$$


where, $R_{\mathrm{i}}$ and $\sigma$ are defined by Eq. (4.8) and (4.27) respectively. The other symbols, which appear in the formula above, follow section 3.2. We correct the guessed at value by Eq. (4.5), so it can be written as

$$
\tau=\tau_{\text {guess }}-\delta \tau, B i=B i_{\text {guess }}-\delta B i, \xi=\xi_{\text {guess }}-\delta \xi
$$

Using these parameters, the system predicts the time/temperature relationship of the remainder process of heating/cooling phases, integrates the sterilization effects and determines an appropriate starting time, $t_{\mathrm{q}}$ of cooling to achieve a desired process effect. From Eq. (3.9), it can be written as

$$
F=\ln (10)\left[\int_{0}^{t_{\mathrm{q}}} \exp \left\{\frac{T-T_{\mathrm{R}}}{Z}\right\} d t+\int_{t_{\mathrm{q}}}^{t_{\mathrm{e}}} \exp \left\{\frac{T-T_{\mathrm{R}}}{Z}\right\} d t\right]_{\mathrm{x}=0}
$$

By numerically integrating the first term in the above equation with a suitable time increment, an upper limit time, $t_{\text {max }}$ can be assumed as the time that $F$ reaches the given goal. The time, $t_{\mathrm{q}}$ to start cooling may be determined through a trial and error procedure between 0 and this upper limit time, $t_{\max }$. At this time, integrating limit, $t_{\mathrm{e}}$ of the second term of the above equation is chosen as the time that center temperature reaches room temperature.

A typical result of experiments (example copy of monitor display) is shown in Fig. 3. The $\times$ marks denote the temperature collected with a sensing probe, 21 points with 10 second intervals. When all the preset number of data were collected, the computer started iterating calculations to estimate $\tau, B i$, and $\xi$. The progress of improvement in approximating parameters $\tau$ and $B i$ was monitored through the display as shown on the right in Fig. 3.

An on-line control system for food freezing In the freezing process, there is a time with steep reduction of the latent heat load. When the density of suction gas is falling, the delivery capacity of the compressor should also be falling, and then liquid refrigerant begins to accumulate in the evaporator. Such accumulation of liquid in an evaporator brings about the hazard known as liquid compression, which is a serious problem of the refrigerator. The feeding rate of refrigerant to the evaporator through the expansion valve must be controlled to a certain limit immediately before the latent heat load decreases. This becomes the system's lifetime extension and the saves of energy consumption, in addition to being an issue of the safety of the refrigeration system.

During the early stages of the freezing process, the system collects a series of time/temperature data via a two sensing probe installed in the food body and in the evaporator.

One technique is using an analytical solution Eq. (3.44). As described in section 4.2, the system analyzes inversely the data to estimate an inserted position, $\xi$ of the sensor, as well as thermal parameters of the food such as Biot number, $B i$ and time constant, $\tau_{\mathrm{f}}$.

This system was tested by using 50 sets of time/surface temperature measurements during the freezing of wheat flour dough $(D=16 \mathrm{~mm})$. One of the test results is shown in Fig. 4.

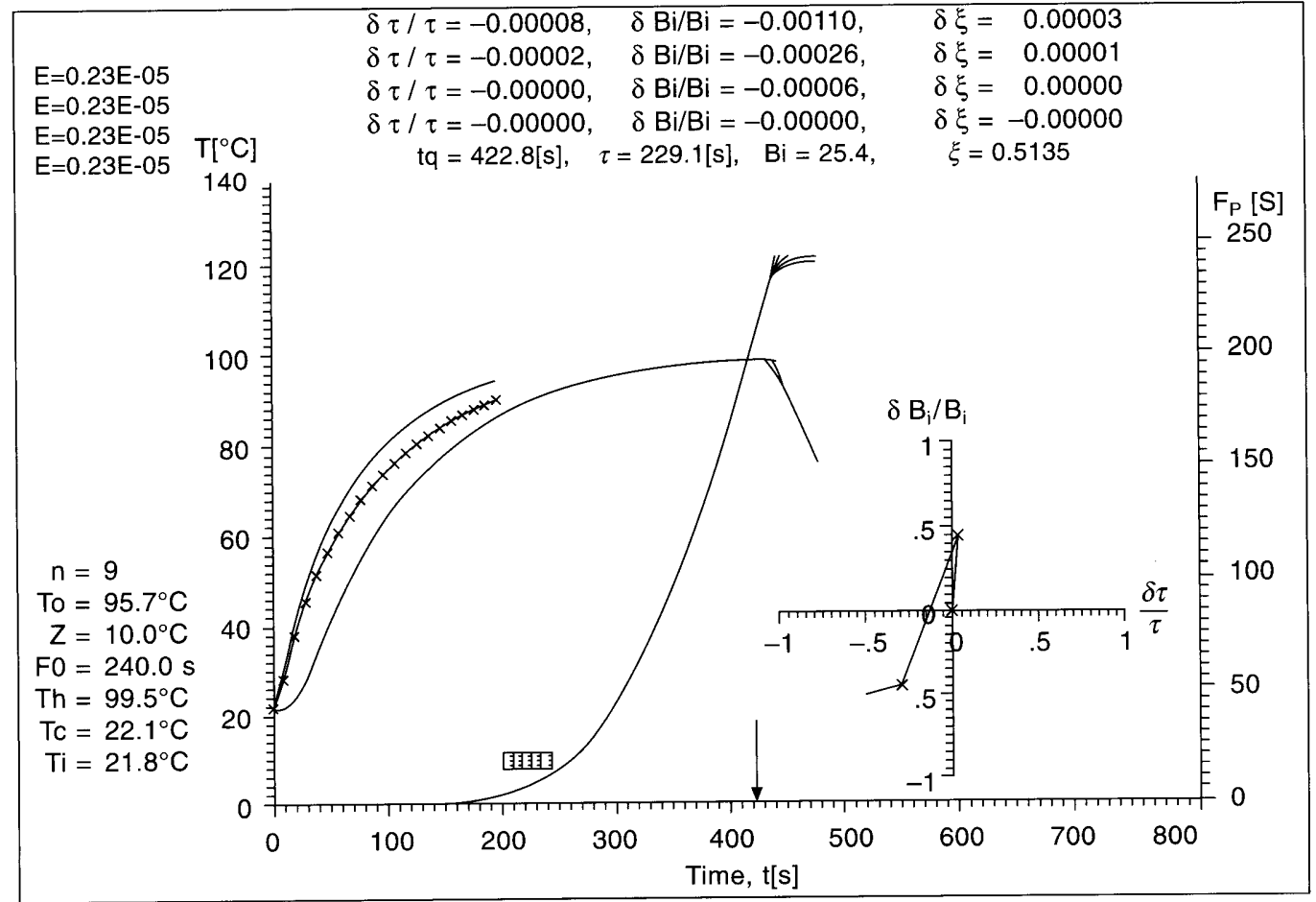

Fig. 3. An example of monitor display. The temperatures collected by computer are denoted by $\times$ marks. The extent of the process in improving parameter approximation is shown on the top. The variance in nonlinear multiple regression, denoted by $E$, is shown at the upper left. The increments parameter approximation is shown on the top. The variance in nonlinear multiple regression, denoted parameters used in the iteration process are plotted on the right. After it computing the estimated parameters, $\tau, B i$, and $\xi$, the computer predicted the temperature history at the geometric center of the sample, $T_{\mathrm{m}}$, and corresponding lethal rate integral, $F_{\mathrm{p}}$, in a trial and error search for the appropriate $t_{\mathrm{q}}$ value. The appropriate $t_{\mathrm{q}}$ finally obtained is indicated by an arrow at the bottom. The striped bar at the bottom denotes the time period used by the computer (NEC-PC98XL $16 \mathrm{MHz}$ ) for calculation. 


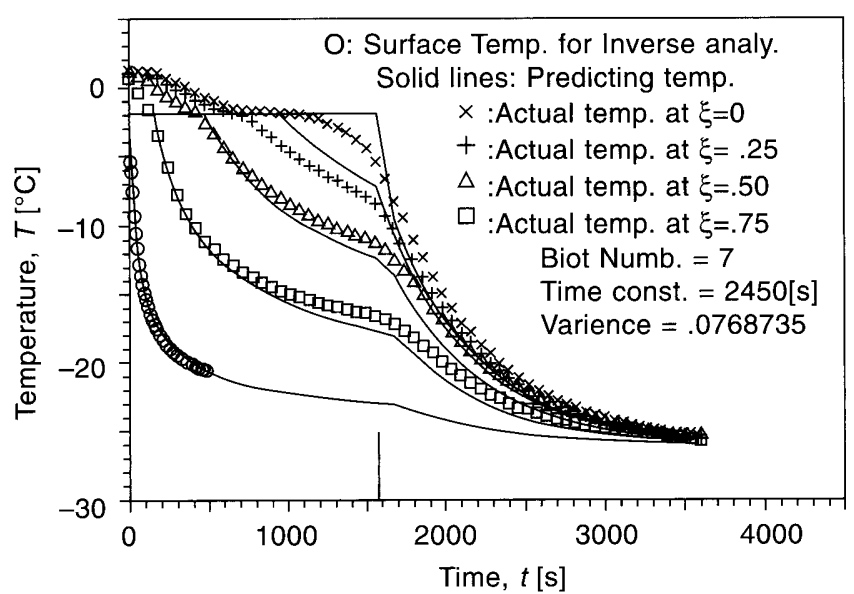

Fig. 4. Freezing curve for wheat flour dough $(D=16 \mathrm{~mm})$.

When the food is not a slab, we apply Eq. (3.46) and we calculate backward five parameters in Eqs. (3.47-48). After the thermal parameters are estimated, the cooling load for the future can be estimated.

\section{Conclusion}

There are many of assumptions and approximations in the exercise introduced in this text. These will become unnecessary if the analysis techniques improve. According to improvements in computer performance, papers too numerous to list hear and many textbooks on the inverse problem have been published. The definitions of the inverse problem are various. The techniques, too, are various. The inverse problem in the food industry is widely present. If the inverse analytical techniques become popular in future, it is the wish of this author that the word "inverse" will disappear from all titles.

\section{References}

Abel, N.H. (1823). Solution de quelques problemes a 1'aide d'integrales definies. Mag. Naturvidenskaberne, Aargang I, Bind 2, Christia, 11-27.
Abel, N.H. (1826). Auflosung einer mechanischen Aufgabe. Crelles J., 153-157.

Beck, J.V., Blackwell, B. and Haji-Sheikh, A. (1996). Comparison of some inverse heat conduction methods using experimental data. Int. J. Heat Mass Transfer, Vol. 39, No. 17, 3649-3657.

Dorai, G.A. and Tortorell, D.A. (1997). Transient inverse heat conduction problem solutions via Newton's method. Int. J. Heat Mass Transfer, Vol. 40, No. 17, 4115-4129.

Ferrari, C. and Tricomi, F.G. (1962). "Aerodinamica Transonica." Edizioni Cremonese, Rome. translated in English by Cramer, R.H., (1968), under the title "Transonic Aerodynamics." Academic Press, New York.

Flach, G.P. and Ozisik, M.N. (1989). Inverse heat conduction problem of simultaneously estimating spatially varying thermal conductivity and heat capacity per unit volume. Numerical Heat Transfer, Part A, vol. 16, 249-266.

Groetsch, C.W. (1993). "Inverse problems in the mathematical science." Vieweg \& Sohn Verlagsgesellschaft, Wiesebanden.

Hildenbrand, P. (1980). An approach to solving the optimal temperature control problem for sterilization of conduction heating foods. J. Food Process Eng., 3, 123-142.

Lesnic, D., Elliott, L, and Ingham, D.B. (1998). The solution of an inverse heat conduction problem subject to the specification of energies. Int. J. Heat and Mass transfer, vol. 41, No. 1, 25-32.

Mihori, T., Siozawa, K., Matuoka, H. Okajima, J. and Sugimoto, T. (1990). Heat conduction in solution dispersed growing fine crystals. Trans. Jpn. Soc. Heat and Fluid Eng., 5, 43-51. (In Japanese).

Mihori, T., Watanabe, H. and Kaneko, S. (1991). A control system for achieving correct heat sterilization processes. J. Food Proc. Preserv., 15, 135-155.

Mihori, T. and Watanabe, H. (1992). A rapid freezing experiment to assess the effect of temperature/position variable thermal conductivity on freezing time estimation. Nippon Suisan Gakkaishi, 58, 29-38.

Mihori, T. and Watanabe, H. (1994a). A two-stage model for on-line estimation of freezing time of food. J. Food Eng., 23, 69-89.

Mihori, T. and Watanabe, H. (1994b). An on-line method for predicting freezing time using time/temperature data collected in the early stages. J. Food Eng., 23, 357-373.

Park, H.M., Chung, O.Y. and Lee, J.H. (1999). On the solution of inverse heat transfer problem using the Karhunen Loeve Galerkin method. Int. J. Heat and Mass Transfer, vol. 42, 127-142.

Sanz, P.D., Ramos, M. and Aguirre-Puente, J. (1999). One stage model of foods freezing. J. Food Eng., 40, 233-239. 